\title{
Stamp Verification for Automated Document Authentication
}

\author{
Barbora Micenková $^{1,2}$, Joost van Beusekom $^{2}$, and Faisal Shafait ${ }^{2}$ \\ 1 Aarhus University, Aarhus, Denmark, \\ 2 German Research Center for Artificial Intelligence (DFKI), Kaiserslautern, \\ Germany \\ Barbora@cs.au.dk, j_v_b@gmx.net, Faisal.Shafait@dfki.de
}

\begin{abstract}
Stamps, along with signatures, can be considered as the most widely used extrinsic security feature in paper documents. In contrast to signatures, however, for stamps little work has been done to automatically verify their authenticity. In this paper, an approach for verification of color stamps is presented. We focus on photocopied stamps as nongenuine stamps. Our previously presented stamp detection method is improved and extended to verify that the stamp is genuine and not a copy. Using a variety of features, a classifier is trained that allows successful separation between genuine stamps and copied stamps. Sensitivity and specificity of up to $95 \%$ could be obtained on a data set that is publicly available.
\end{abstract}

\section{Introduction}

Stamps are still widely used in everyday paper-based business correspondence. They can be frequently found on invoices and official documents. The widespread use of high-quality color copiers, however, has made it feasible to generate forged documents with stamp images that can be easily mistaken for genuine stamps.

A trend that is aggravating the problem is, that more and more companies are scanning all their incoming mail in order to automate their processing as far as possible. An implicit first line inspection [1] formerly done by employees who visually checked the documents is disappearing. However, in state-of-the-art document digitization systems, as they are used in many companies nowadays, absolutely no verification of authenticity of the document is done. Thus, even simple forgeries can easily be accepted as genuine documents, as long as the system is able to read and understand its content.

To cope with these problems, automatic authentication tools are needed to substitute the former first line inspection done by employees. Several approaches exist in literature that use intrinsic features (features that originated in a normal document generation process) for authentication of the document source. These approaches follow the idea of comparing an incoming ("questioned") document with genuine documents from the same source that are already in the database. If the features differ significantly, the incoming document is considered as suspicious, and further examination by an expert can be initiated. 
Apart from using intrinsic features, also extrinsic features (features that are added solely to allow authentication of the document) can be analyzed, as e.g. signatures, counterfeit protection system (CPS) codes and stamps. For signatures (e.g. [2]) and CPS codes [3], automatic approaches have been presented in literature. For stamps, however, no automatic approach for ensuring their genuineness has been presented so far, to the best of our knowledge.

In order to verify the genuineness of a stamp ${ }^{3}$, the stamp first has to be extracted from a document image. The question that arises is, how a stamp can be automatically identified and, in what ways it differs from other elements in the document image, e.g. logos. Specific positions, shapes and colors have already previously been used to detect stamps. However, considering documents with e.g. logos or other graphical content, these features alone will not lead to good stamp detection. Other features have to be added that better describe the essence of a stamp imprint, namely the very specific way of imprinting a stamp. It becomes clear, that stamp detection and stamp verification are two closely related and partially overlapping problems.

Therefore, our previously presented method for stamp detection [4] is improved and extended to enable also stamp verification. A two stage approach is followed: first, stamps are detected and extracted based on the approach presented in [4]. The resulting stamp candidates are then subjected to a second classifier trained to discriminate between genuine stamps and copied stamps.

An overview of the system is given in Figure 1. Using color space transformations and $k$-means clustering, the scanned color image is split according to colors. Using the XY-cut algorithm [5], the separated colors are segmented into stamp candidates. Finally, different features are extracted which are then used to classify the candidates into genuine stamps and non-stamp objects (laser-printed or photocopied). The detection and verification parts can also be separated if the application scenario requires it.

The remaining sections of the work are organized as follows: Section 2 gives an overview of the advances in stamp detection and verification. Section 3 describes our overall approach. Evaluation and results are presented in Section 4 and 5 . The paper concludes with Section 6 .

\section{Related Work}

Concerning the main goal of the proposed work, stamp verification, no relevant literature could be found by the authors among standard document image processing publications. However, some work has been done in related fields.

Concerning stamp detection, or localization of stamps in document images, a reliable general solution has also not been given yet [6]. As prior knowledge of the structure (shape or color) of the stamp is helpful to localize it in images, previous research has been focused on detection of stamps of a particular type. The approaches selected by various authors can be divided into three groups:

\footnotetext{
${ }^{3}$ In this work, we mainly focus on rubber stamps
} 


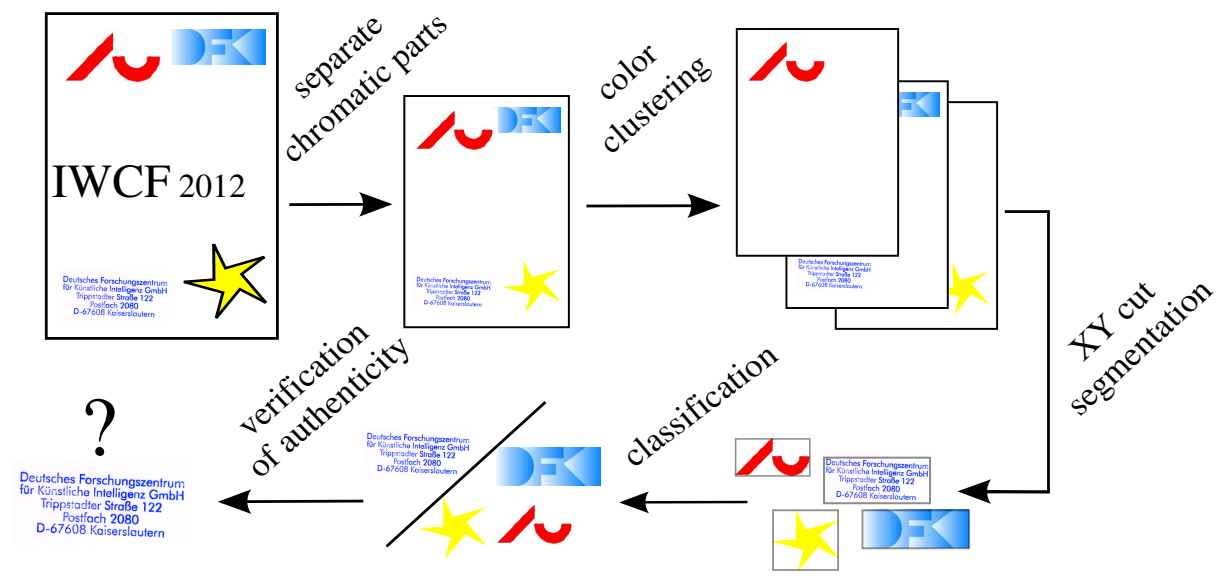

Fig. 1. An overview of the stamp detection and verification system.

(1) Color analysis approaches: these methods assume that the stamp is of a predefined color. Objects of this color are then detected by the methods and returned as stamps. Examples are [7-9], that use color clustering in the RGB color space to detect stamps of a specific color. Forczmański et al. [6] presented a method that uses color clustering in the $Y C_{b} C_{r}$ color space to detect red and blue stamps.

(2) Shape information approaches: these approaches assume that the stamp has a known, fixed shape, e.g. oval or rectangular. In [10], Chen, Liu et al. detect seals on Chinese bank checks with a region-growing algorithm. They suppose the seal to be the only object in the cheque to have an outer frame. Zhu et al. [11] presented a method that uses Hough Transform [12] to search for circular and elliptical candidates for stamps.

(3) Symbol spotting approaches: if the images of the stamps are known beforehand, symbol spotting techniques can be used for stamp recognition. Symbol spotting is a way how to efficiently localize symbols in images, without need of previous segmentation of the image [13]. These techniques are more suitable for stamp retrieval than for general detection as they are initiated with a query image selected by user (QBE - query by example). The query is used to find similar symbols in the database. Examples of such approaches can be found in [14]. Symbol spotting approaches could be used to distinguish between known stamps and forged stamps with the same content but different layout. For copied stamps, however, symbol spotting techniques will fail to classify them as non-genuine stamps.

Printing technique recognition can also be considered as a related field, since stamps are a special form of printing. Ink versus laser print classification [15], ink versus laser versus photocopy classification [16], or laser versus photocopy [17] are a few examples of such methods. However, to the best of the 
authors' knowledge, no method has been presented so far for detecting stamps based on their printing technique features.

A relevant method proposed by Berger et al. [18] can separate overlapping objects of a very similar color (e.g. stamp and signature) by means of support vector machines, Although good results are obtained by this approach, it is not applicable in our settings, since small areas belonging to each object have to be chosen manually.

\section{Method Description}

In this paper we consider stamps to be single-color (blue, green, red, ...) objects. Therefore, the basic idea of how to detect them in an image is to group components having the same color and being close to each other at the same time. Special cases of multiple-color stamps (such as in Fig. 7) are detected as multiple stamps and then merged.

With such an approach we can determine candidate segments. Each stamp in the image forms one candidate segment. If there are color logos or pictures present in the image, they (or their single-color parts) are also identified as candidate segments. In the next step, the challenge is to classify which segments represent genuine stamps and which correspond to printed objects such as copied stamps or logos (Section 3.2 and 3.3). Segmentation by color clustering and extraction of candidate segments has been described in our previous work [4]. Therefore, only a short revision will be given in Section 3.1.

\subsection{Segmentation}

The $R G B$ color model is not convenient for image segmentation because of high correlation among the channels [19]. To process color stamps, it is desirable to first separate out background, black text and other approximately achromatic (white, black and grey) parts of the image. For this purpose, $Y C_{b} C_{r}$ color space is useful because it separates information about luminance $(Y)$ and chrominance $\left(C_{b}, C_{r}\right) . C_{b}$ corresponds to blue and $C_{r}$ to red difference.

To separate out pixels close to grey levels, projection on $C_{b} C_{r}$ plane is made and each pixel value is treated as a polar vector $(r, \theta)$, where $r=\sqrt{C_{b}^{2}+C_{r}^{2}}$ and $\theta=\operatorname{atan} 2\left(C_{b}, C_{r}\right), \theta \in[0,2 \pi)$. A threshold $T$ is fixed and all vectors with magnitude $r>T$ are marked as chromatic. These are used for color clustering.

Color Clustering is needed for separation of components of different colors. From 3D scatter plots in $Y C_{b} C_{r}$ color space it can be observed that clusters corresponding to inks always have elongated shapes and that they stretch from the background cluster. A good separation can be done by projecting the pixel vectors onto $C_{b} C_{r}$ plane and taking just the polar vector $\theta$ as the discriminative property. The angle values are quantized into 360 bins and a histogram is created. The $k$-means clustering algorithm is then run on the (1D) angle histogram space 
which is very fast. Number of clusters is estimated by an approximate calculation of number of peaks in the histogram.

The result of color clustering are $k$ binary mask images of the same size as the original. Black pixels denote parts of the original image having similar color and belonging to one color cluster.

Candidate Segments have to be extracted out of the mask images. Since there can be more objects of the same color on the page, the mask images have to be segmented. However, one stamp is usually composed of several connected components, therefore connected components labeling is not suitable for this task. Instead, the XY-Cut algorithm [5] is used. It recursively partitions the page until it returns minimum bounding boxes for the candidate segments.

\subsection{Feature Extraction}

To differentiate stamp candidates from printed objects such as logos, color texts and potentially also copied stamps, classification must be performed. From each identified candidate segment, features are extracted. The features relate to geometrical properties of the segment, its color hue and the quality of the print.

Geometrical and color features for stamp detection were described in our previous work [4]. Briefly, it is width-to-height ratio, area of the minimum bounding box, pixel density within the bounding box, rotation and standard deviation of hue. Rotation is computed in case that some text-lines are detected.

Standard deviation of hue can be well exploited also to distinguish between genuine and copied stamps. Variation of hue within the imprint is significantly greater for copied stamps than for genuine ones. An illustration is given in Fig. 2. Besides, we propose new features related to the quality of the print since stamp imprints have several remarkable properties.

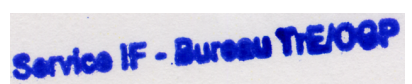

(a) Genuine stamp

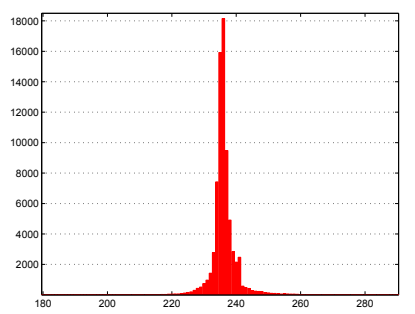

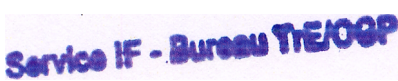

(b) Copied stamp

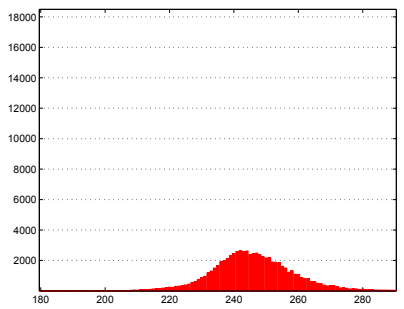

Fig. 2. Histograms reveal that the variation in hue is greater for the copied stamp. 
Uniformity of Area. Stamp imprints can be perceived as uniform, having no distinct texture, as opposed to laser-printed or photocopied objects. Printers (and copiers) cause frequent alternations in intensities - a pattern which is well visible in large single-color areas after magnification.

Lampert et al. [15] worked on recognition of source of a printed text (inkjet, laser printer or copier). They used a technique similar to co-occurrence matrices [20] but they computed them from two different images. The first was the original image and the second a certain transformation of the original. We adopted this technique for feature extraction and selected Gaussian smoothing for transformation of the input image.

Co-occurrence matrix $H(i, j)$ of a grayscale image $f(x, y)$ and its smoothed version $s(x, y)$ can be mathematically defined as

$$
H(i, j)=\frac{1}{M N} \sum_{m=1}^{M} \sum_{n=1}^{N} \begin{cases}1 & \text { if } f(m, n)=i \text { and } s(m, n)=j \\ 0 & \text { otherwise. }\end{cases}
$$

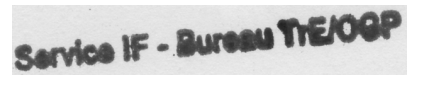

(a) Genuine stamp (8-bit)

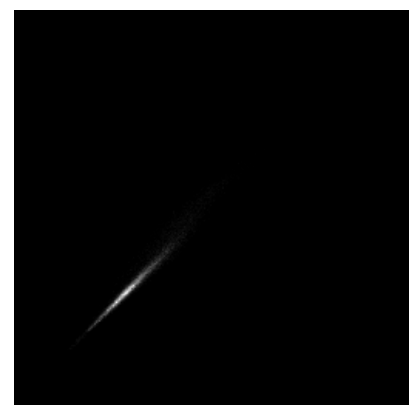

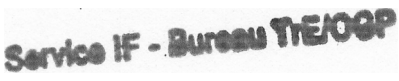

(b) Copied stamp (8-bit)

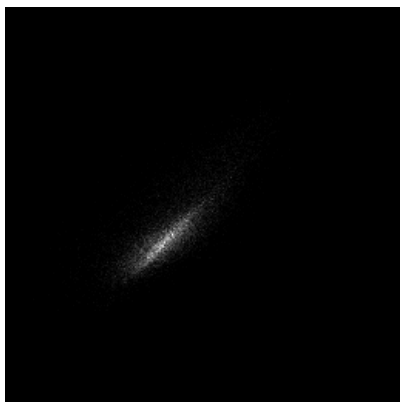

Fig. 3. Visualization of modified co-occurrence histograms of two subjects - a genuine stamp and its copy (the greater values the brighter). It is clear that in the $2 \mathrm{D}$ histogram of copied stamp, values are more scattered whereas in the histogram of genuine stamp, the major mass is on the diagonal. Note that only the area of stamps without background was used for computations.

Although the definition is given in terms of images, we are actually working with particular regions of interest (i.e. only the pixels belonging to the candidate segment). The matrix is a $2 \mathrm{D}$ histogram expressing how often a value $i$ in the original image occurs in combination with a value $j$ in the transformed image. For illustration, 2D histograms of a genuine and a copied stamp from Fig. 2 are visualized in Fig. 3.

The histogram itself is too big and sparse to give us itself a meaningful information about the texture. Therefore a subset of four so called Haralick 
features [20] is extracted from it: contrast, correlation, energy and homogeneity. Please find the formulas in [15].

Sharpness of Edges. Edge sharpness is the degree of intensity change at a particular region in the image and it can be measured by gradients. Comparing gradient maps of genuine stamp imprints and copied or laser-printed text characters, it can be observed that genuine stamp imprints are characterized by slightly higher gradient values. It is caused by the fact that stamps tend to have more blurred and smooth edges. An example of gradient maps of a genuine and a copied stamp is given in Fig. 4.
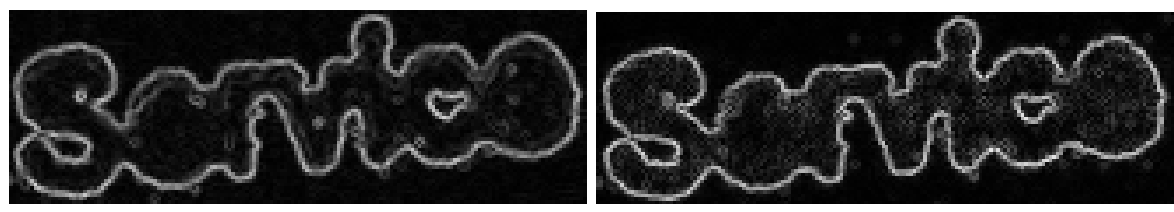

Fig. 4. Magnitude gradient images of genuine (left) and copied (right) stamp. Gradient values at the edges of copied stamp are slightly higher than for genuine stamp.

The gradient of an intensity function $f$ at each coordinates $(x, y)$ is a $2 \mathrm{D}$ vector $\nabla \mathbf{f}=\left(G_{x}, G_{y}\right)$ where $G_{x}$ and $G_{y}$ are derivatives in the horizontal and vertical direction. The gradient vector points in the direction of the largest intensity increase. The magnitude of this vector is given by

$$
\nabla f=\left[\left(\frac{\partial f}{\partial x}\right)^{2}+\left(\frac{\partial f}{\partial y}\right)^{2}\right]^{1 / 2}
$$

To obtain approximate gradient images, the original image must be converted to grayscale and then convolved e.g. with Prewitt filter. To extract statistical information needed for classification, the magnitude gradient image $\nabla f$ is computed and subsequently its histogram $\nabla H(i), i=0, \ldots, 255$ is constructed.

Schreyer et al. [21] analyzed gradient histograms of text characters to differentiate the printing technique (laser print, inkjet print or photocopy). According to the study, the greatest variance among different printing techniques can be observed in the histogram in intervals $[1,40]$ and $[80,120]$.

As statistical features for classification, the mean, standard deviation and maximum are derived from the appropriate intervals of the histogram.

Roughness of Edges. Due to the ink diffusion effect, stamp edges are rough as opposed to edges of laser-printed characters.

Schulze et al. [22] have shown that edge roughness features are useful for recognition of the source of printed text. A method how to measure edge roughness is to compare a binarized image to its smoothed version and then derive features via distance mapping. 
Distance mapping [23] requires the input image as well as the smoothed input image to be binarized. Then, the distance map is initialized with values from $I_{b i n}^{\text {smooth }}$ and then distances of all foreground pixels of $I_{b i n}$ are propagated to the nearest background pixel of $I_{b i n}^{s m o o t h}$. Denoting background pixels by 0 , for each entry $(\mathrm{x}, \mathrm{y})$ of the distance map we get:

$$
D(x, y)=\min \left\{d: d=\sqrt{(x-m)^{2}+(y-n)^{2}}, I_{b i n}^{\text {smooth }}(m, n)=0, \forall m, n\right\} .
$$

Only edge pixels of $I_{b}$ are of interest and so their distances are extracted from the map and a histogram is created. Statistical measures such as mean, standard deviation and maximal and relative distance are then derived.

\subsection{Classification}

For both tasks, detection and verification of authenticity, classification is performed. In the first stage we want to differentiate between stamps and other objects in the page. In the second stage, only segments determined as stamps are further processed and the genuine ones must be distinguished from copies. The schema of two-stage classification is given in Fig. 5 .

Before actual classification, candidates whose geometrical features exceed fixed thresholds are removed. The features are: width-to-height ratio, area and number of pixels within the bounding box and pixel density. The thresholds are set loose enough to eliminate only extreme candidates.

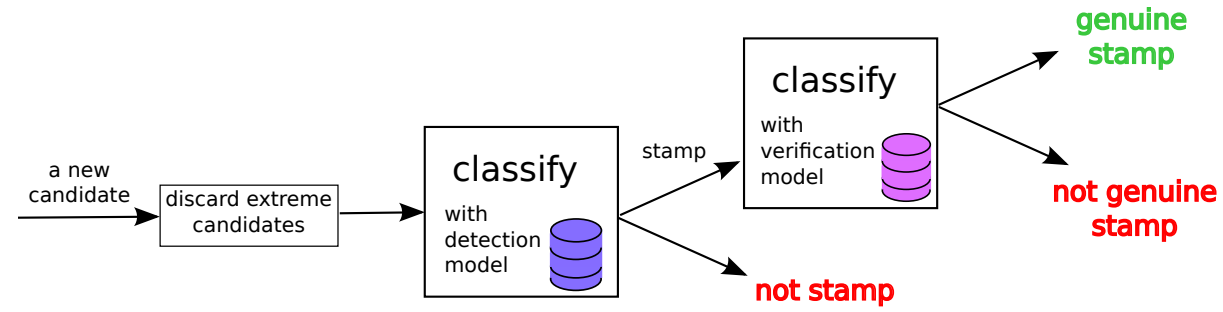

Fig. 5. Two-stage classification for joint stamp detection and verification.

Training of the full system is then also performed in two phases (see Fig. 6). A model for detection and a model for verification are trained separately. There are altogether 13 features used for detection: rotation, standard deviation of hue and all print-related features. Our experiments with verification indicated that the edge roughness features ( 4 features) do not improve the classification result. Also rotation is naturally not relevant for verification ( 1 feature), and so the remaining 8 features are used.

It has to be noted, that in the first stage where stamps are separated from non-stamp candidates, very obvious stamp copies might be already revealed 


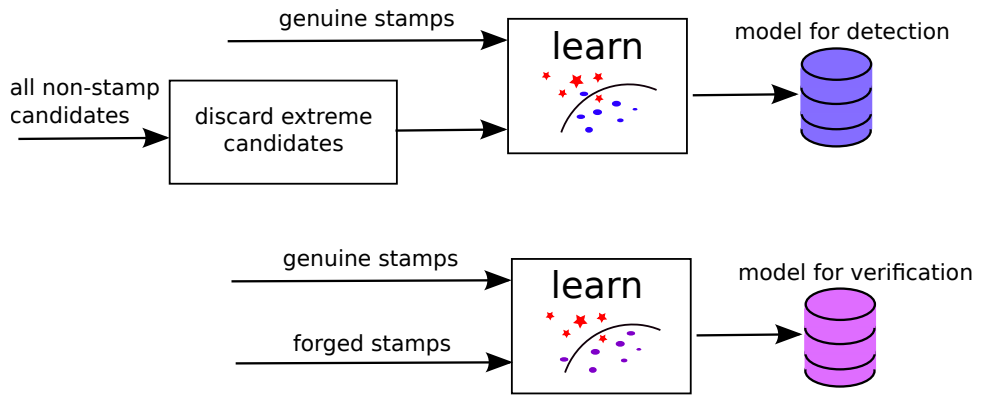

Fig. 6. Training for the two-stage classification.

because their properties are close to properties of other printed objects such as logos.

Different classifiers have been tested for the two stages: Multi-Layer Perceptrons, Random Forests, Bayes Networks and Support Vector Machines (SVM). It showed that the best results were achieved using an SVM classifier.

\section{Evaluation}

We evaluated both classification stages, detection and verification, separately. In [4] we published a data set of 400 document pages for evaluation of stamp detection algorithms. This data set contains stamped invoices with color logos and texts. Stamps are often overlapped with text or other objects. It was generated by printing automatically generated invoices, stamping them manually and scanning them in color with a resolution of $600 \mathrm{dpi}$. To limit the effort of ground-truth labeling, the lower resolutions were obtained by downscaling.

To evaluate the performance of verification, we created and published a new data set ${ }^{4}$ of copied documents. A total of 14 invoices were selected randomly from our data set and their copies were made on 5 different models of Ricoh Aficio copy machines. We obtained 70 images with 78 copied stamps altogether.

To discover the ideal settings for SVM classifiers, a grid search was used to find the parameters which gained the best accuracy. The settings differed a bit for different resolutions of the images but we always used $\nu$-SVC classifier with radial-basis function, $\nu \in[0.08,0.12]$, and $\gamma=\frac{1}{\text { numberOfFeatures }}$.

Stamp detection was evaluated on the 400-page data set scanned at different resolutions by cross-validation with leave-one-out method. In each round, one document page (not a segment) was left out. Altogether training was performed on around 1000 candidate segments, from which 290 were genuine stamps.

Stamp verification was evaluated by 10 -fold cross validation on all available genuine stamps and 78 copied stamps.

\footnotetext{
${ }^{4}$ The data set is available at http://madm.dfki.de/downloads-ds-staver.
} 


\section{Results}

Results of stamp detection in images of different resolutions are given in terms of pixel accuracy (see Tab.1). Recall is the proportion of correctly detected stamp pixels to all stamp pixels in the image while precision is the proportion of correctly detected stamp pixels to all detected pixels. Pixel-wise measures give us a clear picture about the performance of the method.

\begin{tabular}{|l|c|c|c|c|}
\hline & 200dpi & 300dpi & 400dpi & 600dpi \\
\hline Recall & 0.89 & 0.89 & 0.89 & 0.89 \\
\hline Precision & 0.90 & 0.90 & 0.92 & 0.90 \\
\hline
\end{tabular}

Table 1. Results of stamp detection algorithm for different resolutions.

It is not meaningful to give the results of stamp authentication as pixel-wise accuracies since already segmented (genuine or copied) stamps are expected as input. We will express the results in terms of sensitivity and specificity. Sensitivity is the proportion of correctly classified copied stamps to all stamps and specificity is the proportion of correctly classified genuine stamps to all stamps.

\begin{tabular}{|l|c|c|c|c|}
\hline & 200dpi & 300dpi & 400dpi & 600dpi \\
\hline Sensitivity & 0.90 & 0.92 & 0.93 & 0.95 \\
\hline Specificity & 0.96 & 0.91 & 0.96 & 0.96 \\
\hline
\end{tabular}

Table 2. Results of stamp authentication (classification between genuine and forged stamps) for different resolutions.

Note that detection as well as verification of authenticity perform well even for images of low resolution which makes it possible to use them in common office environments. With increasing resolution, more copied stamps are revealed while stamp detection gives stable results for all resolutions. There are also some unanticipated deviations in the values which were probably caused by inappropriate settings of variables dependant on resolution (e.g. radius of masks).

\section{Conclusion}

In this paper we presented the first automatic approach to verification of authenticity of stamps in documents by assuring that they are not photocopies. Having a questioned stamp as input, sensitivity and specificity of $95 \%$ have been achieved on the data set that we created and made publicly available.

Besides, we improved the accuracy of our previously published method on stamp detection by extracting new features that attempt to describe the quality of the print of the candidate segments. We exploited the fact that both tasks, stamp detection and verification of authenticity, have a similar nature because in both cases we intend to differentiate objects printed from a different source.

Combining both methods we have proposed a two-stage approach which can be used as a part of a system for automatic athentication of the document source. 
New incoming documents can be compared to genuine documents from the same source that are already stored in the database, and a missing or photocopied stamp indicates a forgery. To verify the content or the shape of the questioned stamp, symbol recognition or image registration methods could be used.

The assumption that stamps are single-color objects does not limit us from detecting multiple-color stamps at all (though they are rare). The stamp is split into single-color parts which are then detected separately. In a next step they are merged again. An example of one stamp from our data set is given in Fig. 7.

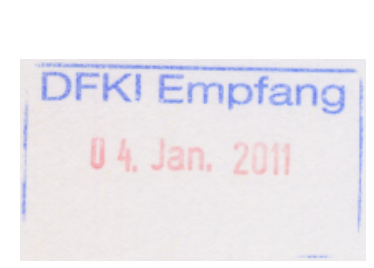

(a) Original stamp

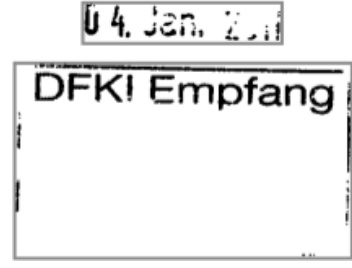

(b) Candidates found

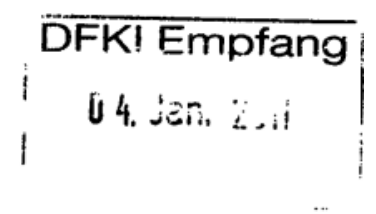

(c) Result

Fig. 7. A two-color stamp is split into two segments during the process and then merged again on output of the stamp detection algorithm.

The presented method works with color parts of documents, therefore detection and verification of authenticity of black stamps is so far not possible. However, segmentation of the achromatic part of the image might also lead to a proper extraction of candidates and then the color-independent features could be used for classification. Such an extension to the method is presumed, but has not been tested yet.

\section{References}

1. van Renesse, R.: Paper based document security - a review. In: European Conf. on Security and Detection, London, UK (April 1997) 75-80

2. Pu, D., Ball, G.R., Srihari, S.N.: A machine learning approach to off-line signature verification using Bayesian inference. In: Proc. of the 3rd Int. Workshop on Computational Forensics. Volume 5718 of Lecture Notes in Computer Science., The Hague, The Netherlands (August 2009) 125-136

3. van Beusekom, J., Schreyer, M., Breuel, T.M.: Automatic counterfeit protection system code classification. In: Proc. of SPIE Media Forensics and Security XII, San Jose, CA, USA (January 2010)

4. Micenková, B., van Beusekom, J.: Stamp detection in color document images. In: Proc. of the 11th Int. Conf. on Document Analysis and Recognition, Beijing, China (September 2011)

5. Nagy, G., Seth, S.C.: Hierarchical representation of optically scanned documents. In: Proc. of the 7th Int. Conf. on Pattern Recognition, Montreal, Canada (July 1984) $347-349$

This project was partially funded by the Rheinland-Palatinate Foundation for Innovation, project AnDruDok (961-38 6261 / 1039). 
6. Forczmański, P., Frejlichowski, D.: Robust stamps detection and classification by means of general shape analysis. In: Proc. of the Int. Conf. on Computer Vision and Graphics, Warsaw, Poland (September 2010) 360-367

7. Ueda, K.: Extraction of signature and seal imprint from bankchecks by using color information. In: Proc. of the 3rd Int. Conf. on Document Analysis and Recognition. Volume 2., Montreal, Canada (August 1995) 665-668

8. Cai, L., Mei, L.: A robust registration and detection method for color seal verification. In: Proc. of the Int. Conf on in Intelligent Computing, Hefei, China (August 2005) 97-106

9. Soria-Frisch, A.: The fuzzy integral for color seal segmentation on document images. In: Proc. of the 13th Int. Conf. on Image Processing, Barcelona, Spain (September 2003) 157-160

10. Chen, L., Liu, T., Chen, J., Zhu, J., Deng, J., Ma, S.: Location algorithm for seal imprints on Chinese bank-checks based on region growing. Optoelectronics Letters 2(2) (2006) 155-157

11. Zhu, G., Jaeger, S., Doermann, D.: A robust stamp detection framework on degraded documents. In: Proc. of SPIE Document Recognition and Retrieval XIII. Volume 6067., San Jose, CA, USA (January 2006) 1-9

12. Tsuji, S., Matsumoto, F.: Detection of ellipses by a modified Hough transformation. IEEE Trans. on Computers 27(8) (1978) 777-781

13. Delalandre, M., Valveny, E., Lladós, J.: Performance evaluation of symbol recognition and spotting systems: An overview. In: Proc. of the 8th IAPR Workshop on Document Analysis Systems, Nara, Japan (September 2008) 497-505

14. Roy, P.P., Pal, U., Lladós, J.: Seal object detection in document images using GHT of local component shapes. In: Proc. of the 25th ACM Symposium on Applied Computing. (March 2010)

15. Lampert, C.H., Mei, L., Breuel, T.M.: Printing technique classification for document counterfeit detection. In: Proc. of the Int. Conf. on Computational Intelligence and Security, Ghuangzhou, China (November 2006) 639-644

16. Schulze, C., Schreyer, M., Stahl, A., Breuel, T.M.: Using DCT features for printing technique and copy detection. In: Proc. of the 5th Int. Conf. on Digital Forensics, Orlando, FL, USA (January 2009) 95-106

17. Tchan, J.: The development of an image analysis system that can detect fraudulent alterations made to printed images. In: Proc. of SPIE Optical Security and Counterfeit Deterrence Techniques V. Volume 5310., San Jose, CA, USA (January 2004) 151-159

18. Berger, C.E., Koeijer, J.A., Glas, W., Madhuizen, H.T.: Color separation in forensic image processing. Journal of Forensic Sciences 51(1) (2006) 100-102

19. Cheng, H.D., Jiang, X.H., Sun, Y., Wang, J.: Color image segmentation: advances and prospects. Pattern Recognition 34(12) (2001) 2259-2281

20. Haralick, R.M., Shanmugan, K., Dinstein, I.: Textural features for image classification. IEEE Transactions on Systems, Man and Cybernetics 3(6) (1973) 610-621

21. Schreyer, M.: Intelligent printing technique recognition and photocopy detection for forensic document examination. In: Proc. of Informatiktage 2009. Volume S-8., Bonn, Germany (2009) 39-42

22. Schulze, C., Schreyer, M., Stahl, A., Breuel, T.M.: Evaluation of graylevel-features for printing technique classification in high-throughput document management systems. In: Proc. of the 2nd Int. Workshop on Computational Forensics. Volume 5158 of LNCS., Washington, DC, USA (August 2008) 35-46

23. Rosenfeld, A., Pfaltz, J.L.: Distance functions on digital pictures. Pattern Recognition 1(1) (1968) 33-61 